\title{
Application of Vessel Wall Magnetic Resonance Imaging in Intracranial Cerebrovascular Pathology
}

\author{
Forrest Hamrick $^{\star}$ (D), Adam de Havenon ${ }^{\dagger}$ (D), Philipp Taussky ${ }^{*}$ (D), Matthew D. Alexander ${ }^{\ddagger}$ (D), J. Scott McNally ${ }^{\ddagger}$ (D), \\ Ramesh Grandhi* (D) \\ Department of Neurosurgery, Clinical Neurosciences Center, University of Utah School of Medicine ${ }^{*}$, Salt Lake City, UT; Department of \\ Neurology, Clinical Neurosciences Center, University of Utah School of Medicine ${ }^{\dagger}$, Salt Lake City, UT; Department of Radiology, University of \\ Utah School of Medicine ${ }^{\ddagger}$, Salt Lake City, UT, USA
}

Vessel wall magnetic resonance imaging (vwMRI) is emerging as an important tool in the diagnosis of cerebrovascular pathology and as an adjunct to guide management and treatment decisions. Unlike lumen imaging, vwMRI yields radiobiologic information by demonstrating abnormalities in the vessel wall structure, atherosclerotic plaque components, and contrast leakage into the arterial wall indicative of endothelial dysfunction. This article will review the most relevant literature relating to vwMRI findings for multiple common cerebrovascular pathologies and highlight its potential role in the management and treatment of patients with complex disease.

\section{J Neurosonol Neuroimag 2019;11(2):105-114}

Key Words: Vessel wall magnetic resonance imaging; Intracranial aneurysm; Intracranial atherosclerosis; Vasculitis

\author{
Received: August 23, 2019 \\ Revised: November 3, 2019 \\ Accepted: November 4, 2019 \\ Address for correspondence: \\ Ramesh Grandhi \\ Department of Neurosurgery, \\ Clinical Neurosciences Center, \\ University of Utah, 175 North \\ Medical Drive, East Salt Lake \\ City, UT 84132, USA \\ Tel: +1-801-581-6908 \\ Fax: $+1-801-581-4385$ \\ E-mail: neuropub@hsc.utah. \\ edu
}

\section{INTRODUCTION}

Intracranial lumen imaging techniques involving noninvasive modalities, such as computed tomography angiography (CTA) and magnetic resonance angiography (MRA), as well as invasive methods, such as digital subtraction angiography (DSA), which are used to screen, identify, and characterize cerebrovascular disease. Using intravenous gadolinium contrast, blood flow suppression, and high-resolution magnetic resonance imaging (MRI) sequences, vessel wall magnetic resonance imaging (vwMRI) can identify vessel wall enhancement, which correlates with inflammation, vessel wall weakening, and mechanical stress. ${ }^{1,2}$

In clinical practice, vwMRI has been used to improve the yield of noninvasive imaging to characterize high-risk features of intracranial vessel pathologies, including intracranial aneurysms (IAs) and intracranial atherosclerotic disease (ICAD), and can aid in the diagnosis of dissection, vasculitis, and reversible cerebral vasoconstriction syndrome (RCVS). Herein, we describe the applications of vwMRI as a clinical tool in the diagnosis and management of patients with cerebrovascular pathologies.

\section{INTRACRANIAL ANEURYSMS}

IAs are characterized by localized structural changes to the arterial vessel wall that lead to weakening and dilation of the vessel wall. Unruptured IAs are present in roughly $3 \%$ of adults, affecting more women than men and increasing in prevalence with age. ${ }^{3}$ The risk of rupture is $1-2 \%$ per year, and risk increases among individuals with hypertension, history of subarachnoid hemorrhage, and aneurysms of large or irregular size and morphology and those with posterior circulation location. ${ }^{4,5}$ Age, ethnicity, family history, cigarette smoking, and geographic location have also been shown to affect the risk of rupture..$^{4-6}$ Given the significant morbidity 
and mortality associated with IA rupture, the identification and treatment of higher-risk unruptured IAs is essential. To date, various scoring systems have been established to identify unruptured IAs with high rupture risk using CTA, MRA, and DSA. Most prominent among these are the earlier subarachnoid hemorrhage, location of aneurysm, age, population, size, and shape (ELAPSS) and population, hypertension, age, size, earlier subarachnoid hemorrhage, site (PHASES) scoring systems, which stratify risk of growth and rupture, respectively. ${ }^{47}$ Despite the inclusion of clinical and aneurysm characteristics, the assessment offered by these systems is incomplete. Certain variables like tobacco use or dome morphology are included in one scale and not the other, while others like dome thrombus or relationship to parent vessels are not included in either. The addition of vwMRI allows identification of aneurysm wall enhancement (Fig. 1), which is a radiobiologic marker indicating aneurysm instability. ${ }^{8}$ This offers the potential to better stratify patients with unruptured aneurysms by risk, both as a tool allowing more detailed analysis of morphological features of an aneurysm and as a marker of inflammation provided by aneurysm enhancement.

Histopathologically, IAs demonstrate loss of the internal elastic lamina, atrophy of the tunica media, and abnormal patterns of extracellular matrix expression. ${ }^{9}$ A number of dynamic and inter-related pathologic pro-
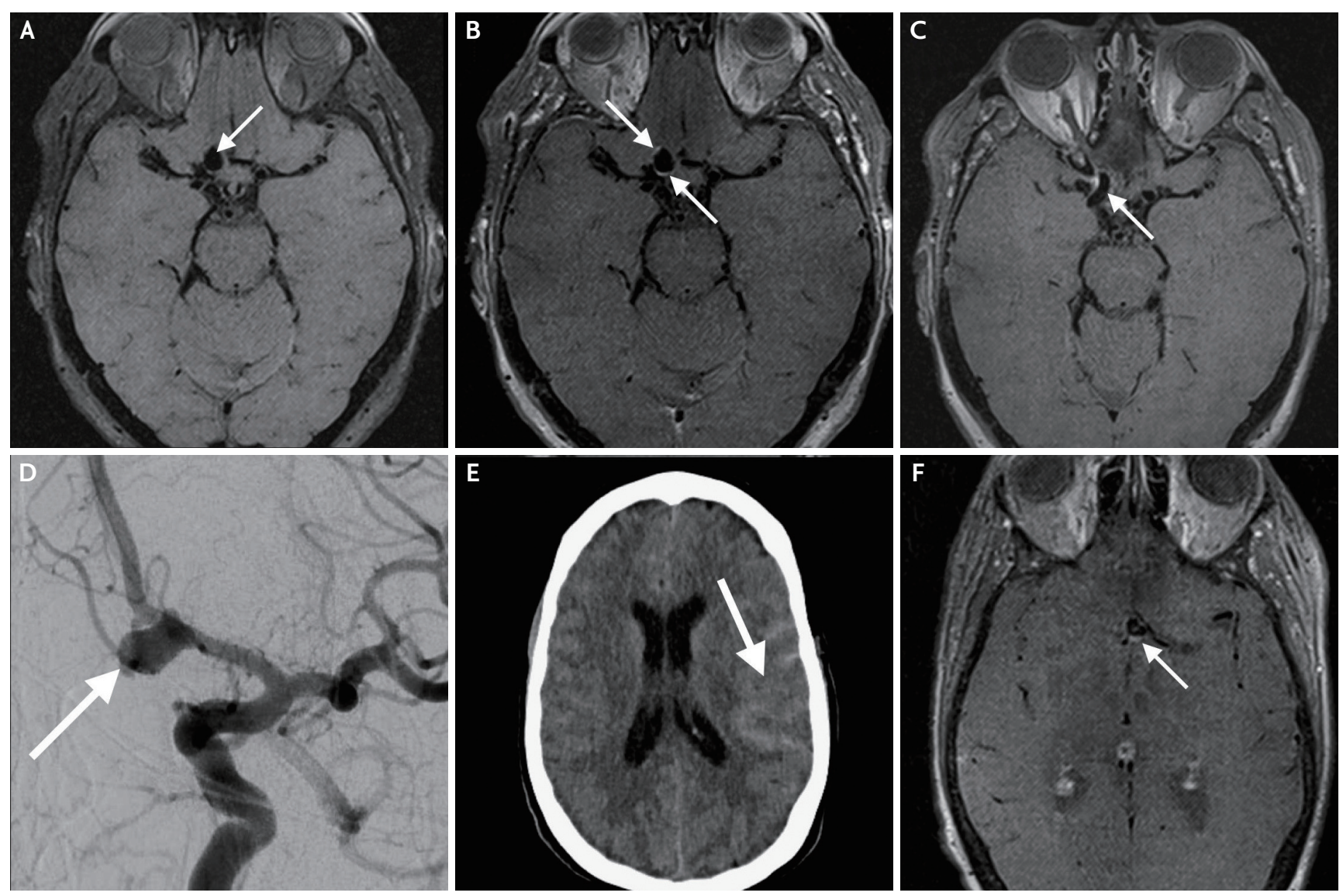

FIG. 1. Aneurysm. (A) 3D axial T1-weighted image showing a large internal carotid artery unruptured aneurysm (white arrow). (B) 3D axial Tr-weighted post-contrast image showing enhancement of the aneurysm wall (white arrows), concerning for active inflammation and instability. (C) $3 \mathrm{D}$ axial T1-weighted post-contrast image after placement of a flow-diverting stent, which has not yet occluded the aneurysm but has created a small area of enhancement remote from the aneurysm (white arrow) that has been reported after flow-diverting stent placement presumed to be related to local inflammation or thrombosis. (D, E) A second patient who presented with thunderclap headache and was found to have an anterior communicating artery aneurysm ( $D$, white arrow, digital subtraction angiogram, lateral projection), which had ruptured and caused subarachnoid hemorrhage ( $E$, white arrow, computed tomography noncontrast, axial). ( $F)_{3} \mathrm{D}$ axial T1-weighted post-contrast image showing enhancement of the aneurysm wall (white arrow), consistent with recent rupture. 
cesses are involved, including endothelial disruption, apoptosis and remodeling of vascular smooth muscle cells within the tunica media, and increased inflammation within aneurysm walls. ${ }^{8,10,11}$ Upregulation of proinflammatory cytokines and molecules, including matrix metalloproteinases, and inflammatory cell infiltration into the aneurysm wall mediate the thinning of the vessel wall, leading to aneurysm growth and rupture. ${ }^{12}$

When compared with the walls of healthy intracranial vessels, the walls of aneurysms can demonstrate increased signal after administration of gadolinium. The mechanism of this finding is debatable, as some research has demonstrated higher wall permeability while other work implicates vasa vasorum, which is not typically present in healthy intracranial arteries but can be found within aneurysm walls, particularly for large aneurysms. ${ }^{13,14}$ The vessel wall enhancement seen in IAs after contrast administration on vwMRI appears to reflect both pathophysiological processes, namely permeability mediated by endothelial dysfunction and inflammation within the aneurysm wall as well as development and proliferation of vasa vasorum. ${ }^{11}$ This association helps explain the tendency for unstable IAs with thinner, more permeable aneurysm walls to enhance more than stable aneurysms. Aneurysm wall permeability—and the resulting degree of enhancementappears to be the best predictor on vwMRI of unstable/ high-risk aneurysms. ${ }^{13}$ In turn, the absence of wall enhancement has been shown to be a significant indicator of a stable aneurysm (negative predictive value: $96 \%$ ). ${ }^{11}$

The current recommendations are for conservative management of smaller aneurysms $(<5 \mathrm{~mm})$ without vessel wall enhancement, because this has been shown to reliably indicates a stable lesion. Enhancement status on vwMRI should be added to the other metrics considered by the ELAPSS and PHASES scores. To best incorporate such data, reproducible quantitative imaging analysis should be employed to promote consistency and reliability when identifying, assessing, and choosing to treat IAs. ${ }^{15}$ At the University of Utah Hospital, recommendations for treating a patient with an intracranial aneurysm are made based on a combination of vwMRI findings, size, and morphology of the IA, clinical symptoms, personal and family history of subarachnoid hemorrhage, and other risk factors such as age, smoking history, ethnicity, and hypertension status.

\section{INTRACRANIAL ATHEROSCLEROTIC DISEASE}

Intracranial atherosclerotic disease (ICAD) poses a significant risk for stroke, accounting for approximately $8 \%$ of strokes in Western countries ${ }^{16}$ and $30-50 \%$ of strokes in patients in Asia. ${ }^{17}$ The prevalence of ICAD is higher in Asians, African Americans, and Hispanics than in North American Caucasians. ${ }^{16}$ There is variation in the morphology of the anterior intracranial circulation among these groups that has been correlated to differences in ICAD localization even when controlling for other risk factors. ${ }^{18}$

Although many ICAD cases are discovered after stroke onset, the asymptomatic incidence is believed to be higher. Common causes of symptomatic ICAD are plaque reorganization and disruption, endothelial damage, inflammation, and immune-related vessel dysfunction. ${ }^{19}$ ICAD is associated with advanced age, diabetes, hypertension, history of stroke, and hyperlipidemia. ${ }^{20}$ ICAD has been traditionally assessed using lumen techniques including transcranial Doppler, MRA, CTA, or DSA. ${ }^{21}$ Analysis of the extent of enhancement and accurate diagnosis of ICAD has been greatly facilitated by the addition of vwMRI to angiographic modalities, especially in intracranial arteries with moderate-to-mild stenosis. ${ }^{22,23}$ Although the eccentric enhancement observed on vwMRI has proven useful in the identification and assessment of ICAD, qualitative interpretation of enhancement varies significantly among reviewers. Our group advocates for quantitative measurement of postcontrast enhancement of target atherosclerotic lesions to improve inter-rater reliability of vwMRI findings. ${ }^{15}$

A hallmark of atherosclerosis involves endothelial cell disruption leading to inflammation and wall remodeling. Intraplaque hemorrhage and plaque enhancement are two of the most important characteristics when identifying and analyzing ICAD. ${ }^{24}$ Atherosclerosis with plaque enhancement on VwMRI has been correlated with a higher risk of thromboembolic complications. ${ }^{19}$ Similar to vessel wall enhancement in unstable IAs, the enhancement seen with ICAD may be the result of an inflammatory process brought on by numerous causes, including disruption of the endothelium or changes to the smooth muscle layer. ${ }^{25,26}$ Further evidence of intraplaque hemorrhage is a relatively reliable marker 
of high-risk ICAD that presents as distinct enhancement on T1-weighted images and signifies a weaker or disrupted plaque. ${ }^{27,28}$ Intraplaque hemorrhage is best detected with heavily $\mathrm{T} 1$-weighted sequences including magnetization-prepared rapid acquisition gradient echo (MPRAGE). ${ }^{29}$ Simultaneous non-contrast angiography and intraplaque hemorrhage imaging can also be used. ${ }^{30}$ To date, multiple meta-analyses in the extracranial carotid literature have focused on the importance of intraplaque hemorrhage for identifying potential stroke sources independent of stenosis ${ }^{31}$ as well as plaques with high future stroke risk. ${ }^{32-34}$ More recently, intraplaque hemorrhage has been found in both low- and high-grade stenotic basilar artery plaques and was independently associated with symptomatic stroke status. ${ }^{35}$ Thus, progression to intraplaque hemorrhage may correspond to a higher risk of plaque disruption and subsequent stroke.

ICAD can result in ischemic strokes via several mechanisms: hypoperfusion from flow-limiting stenosis, occlusion of a perforator leading to a lacunar infarct, thrombosis/embolism from plaque rupture, or a combination of processes. Putatively, vwMRI can help improve diagnosis and management of patients with ICAD by quantifying stenosis, identifying plaque in the region of perforators implicated in lacunar infarction,
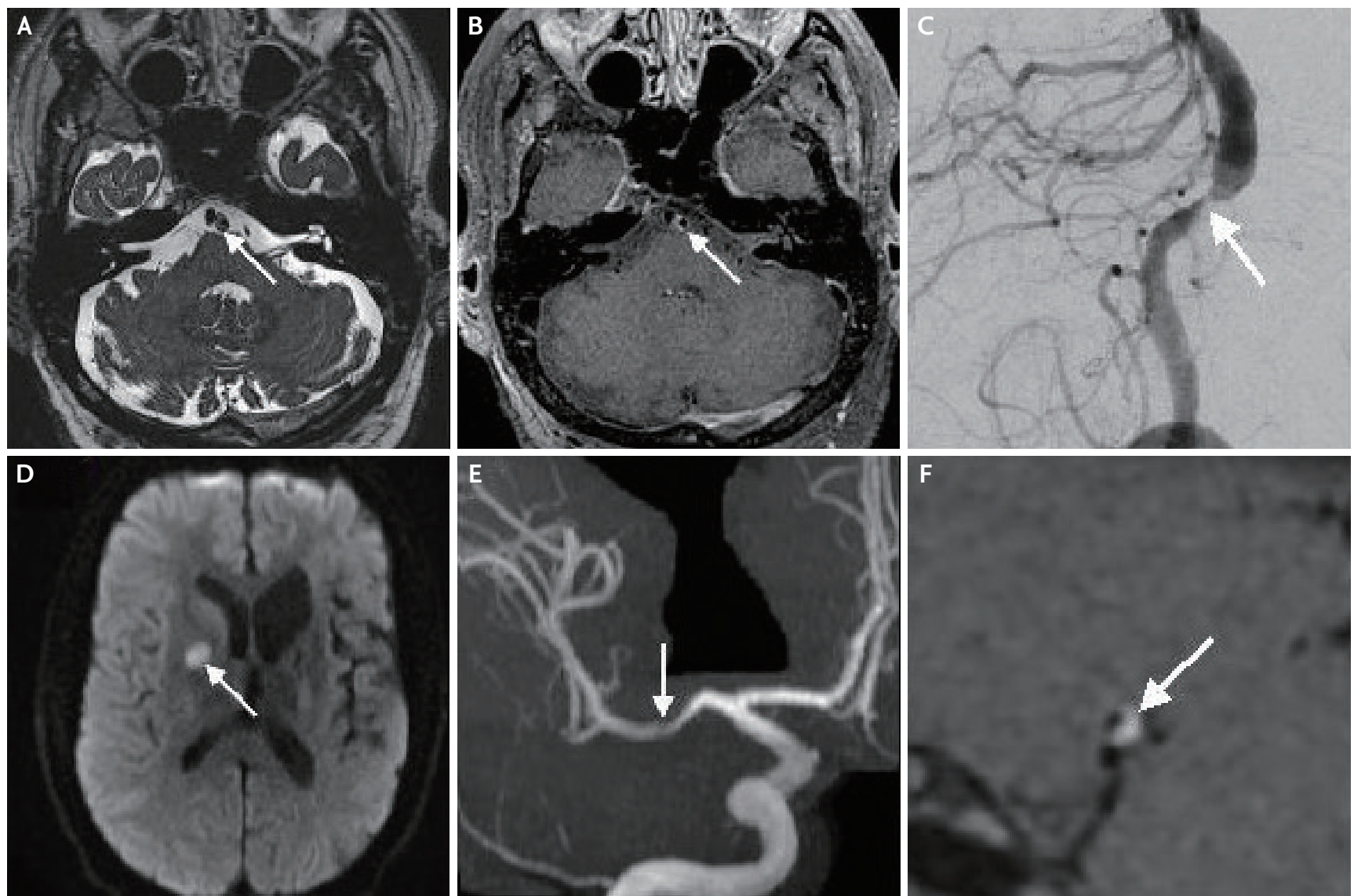

FIG. 2. Atherosclerotic plaque. (A) Atherosclerotic plaque (white arrow) of the vertebrobasilar junction on 3D axial T2-weighted image in a patient who presented with a transient ischemic attack. The fibrous cap appears as the juxtaluminal $T_{2}$ hyperintensity. (B) $3 \mathrm{D}$ axial $\mathrm{T}_{1}$-weighted post-contrast showing eccentric vessel wall enhancement (white arrow), consistent with a recently symptomatic atherosclerotic plaque, and outward remodeling of the plaque. (C) Digital subtraction angiography of the same patient in A and B, showing a lateral view of the atherosclerotic stenosis at the vertebrobasilar junction (white arrow). (D) Diffusion-weighted image and apparent diffusion coefficient axial images of a second patient who presented with left-sided weakness and was found to have acute ischemic stroke in the right internal capsule (white arrow). (E) Time-of-flight magnetic resonance angiography shows minimal right Mi stenosis (white arrow) of the middle cerebral artery, at the origin of the lenticulostriate perforators that supplied the distribution of the ischemic stroke. (F) Sagittal reconstruction of the same right $M_{1}$ segment again demonstrates eccentric wall enhancement (white arrow), consistent with recently symptomatic atherosclerotic plaque, and outward remodeling at the site of the enhancement. 
or demonstrating enhancement indicative of plaque instability and risk for disruption and thromboembolism (Fig. 2).

\section{INTRACRANIAL ARTERIAL DISSECTION}

Intracranial arterial dissection frequently occurs after an initial cervical vertebral artery or cervical internal carotid artery (ICA) dissection but may result from an unrelated intracranial pathology. ${ }^{2}$ Dissections most commonly involve the dorsal wall of the supraclinoid ICA, horizontal (M1) segment of the middle cerebral artery (MCA), and intracranial ( $\left.\mathrm{V}_{4}\right)$ segment of the vertebral arteries. ${ }^{36}$ Intracranial dissections are rare and may cause stroke or subarachnoid hemorrhage, especially in young adults aged $20-50$ years. ${ }^{37}$ Dissections may be further categorized as subintimal or subadventitial, each with distinct clinical course and risks. ${ }^{38}$ Subintimal dissections are more likely to progress to a thromboembolic ischemic event whereas subadventitial dissections are more likely to cause subarachnoid
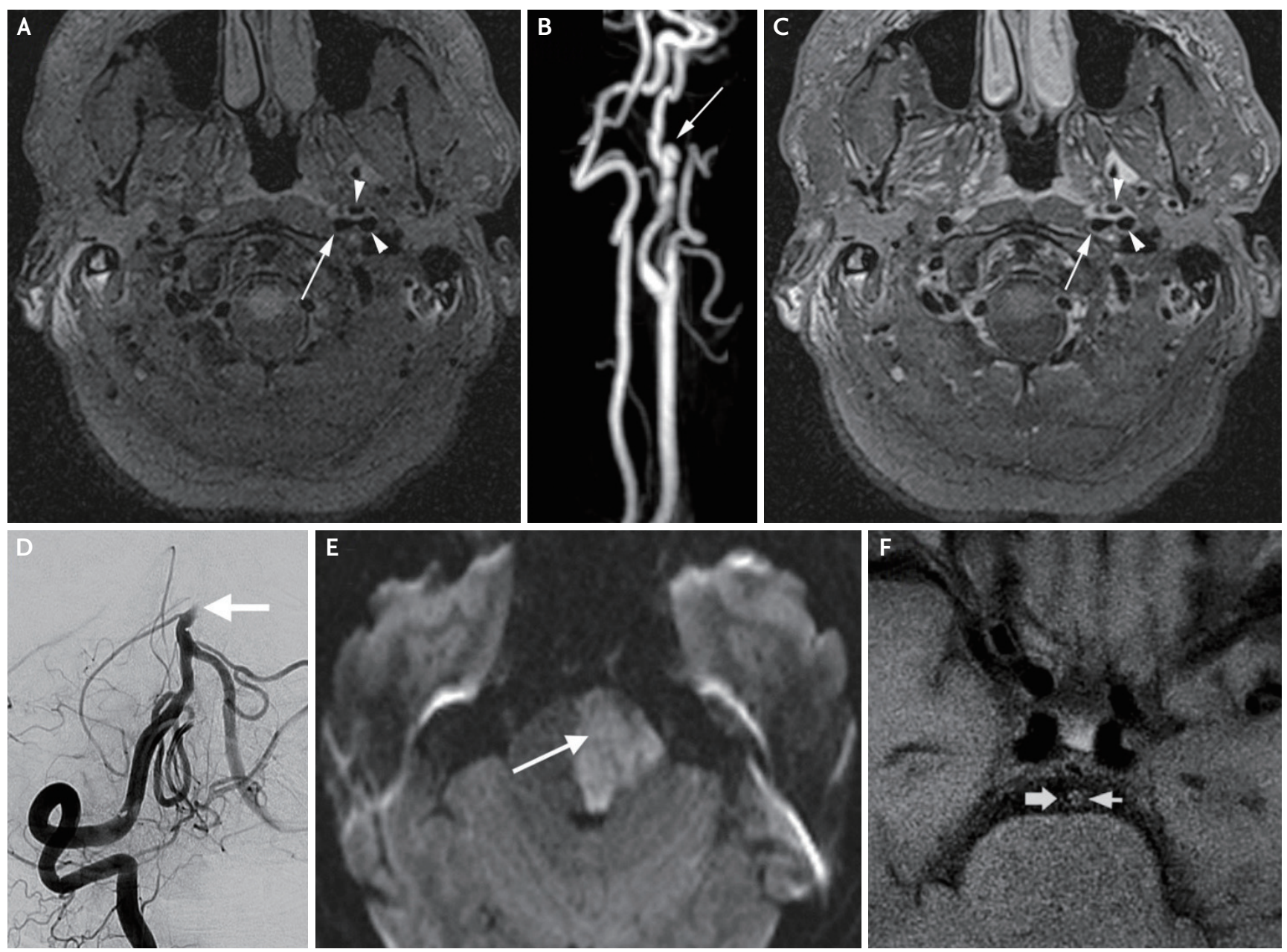

FIG. 3. Dissection. (A) Pre-contrast delay alternating with nutation for tailored excitation (DANTE) image of spontaneous left cervical internal carotid artery (ICA) dissection. Arrow indicates the true lumen of the vessel. Arrowheads indicate the two lobules of the pseudoaneurysm resulting from vessel wall dissection. The wall can be seen between these structures. (B) Magnetic resonance angiography processed by maximum intensity projection (MRA MIP) of the neck reveals the location of dissection and the arrow delineates the associated pseudoaneurysm. (C) Post-contrast DANTE image of spontaneous left cervical ICA dissection. Arrow points to the original lumen. Arrowheads identify two lobules of the pseudoaneurysm. The enhanced vessel wall appears clearly between these structures. (D) Lateral digital subtraction angiography (DSA) showing a basilar artery dissection and near occlusion (white arrow). (E) Axial diffusion-weighted image showing a pontine ischemic stroke (white arrow) from the dissection. (F) $3 \mathrm{D}$ axial $\mathrm{T}_{1}$-weighted image, proximal to occlusion, showing eccentric wall thickening with $\mathrm{T}_{1}$ hyperintense signal (short white arrow) representing arterial dissection, with the remaining patent lumen (thick arrow). 
hemorrhage or lead to pseudoaneurysm formation. Intracranial subadventitial dissections may be harder to detect on imaging because they are generally not accompanied by lumen narrowing or blood flow interference. ${ }^{38,39}$ In contrast, subintimal dissections tend to directly affect lumen size, facilitating identification. ${ }^{1,38}$

On imaging, intracranial arterial dissections may be identified by a double lumen and/or intimal flap, and often the appearance of the dissected vessel will change on follow-up imaging. On MRI, the intimal flap that forms the divide between true and false vessel lumen appears as a curvilinear hyperintensity on $\mathrm{T} 2$-weighted images. ${ }^{2}$ Eccentric wall thickening may also present on imaging and may be tied to inflammation, enhanced vasa vasorum, or reduced blood flow. ${ }^{24}$ An intramural hematoma is a defining feature on MRI and will clearly display blood outside of the true lumen, with heavily T1-weighted sequences including MPRAGE representing the optimal means to visualize hematoma. ${ }^{31,36,38}$ Furthermore, the presence of intramural hematoma signifies a dissection that is more likely to progress to an ischemic event, result in subarachnoid hemorrhage, or lead to the development of a pseudoaneurysm..$^{\circ}$

In the context of intracranial arterial dissection, high-resolution vwMRI sequences may allow more sensitive detection of dissections compared with angiographic imaging alone. ${ }^{2,41}$ Postcontrast vwMRI sequenc- es may facilitate diagnosis because the dissection flap may eccentrically enhance within the dissection (Fig. 3). Despite being the gold standard and highest resolution means of diagnosing intracranial pathology, even DSA may not adequately resolve some high-risk features of a dissection because of its reliance on lumen findings. Therefore, in difficult cases, we recommend that DSA should be supplemented with vwMRI as a means of solidifying a diagnosis.

\section{VASCULITIS AND REVERSIBLE CEREBRAL VASOCONSTRICTION SYNDROME}

Vasculitis refers to a nonspecific inflammatory process within the walls of a blood vessel. Primary central nervous system (CNS) vasculitis is rare and predominantly involves small and medium-sized arteries. Secondary CNS vasculitis is brought on by autoimmune or infectious causes. In either case, vwMRI will demonstrate smooth, concentric, relatively homogenous enhancement of the thickened vessel walls (Fig. 4). As in the case of many of the previously mentioned cerebrovascular pathologies, the inflammatory processes that occur in the wall of the arteries involved may lead to greater permeability to contrast agent. In patients with multiple ischemic strokes in diverse areas of the brain
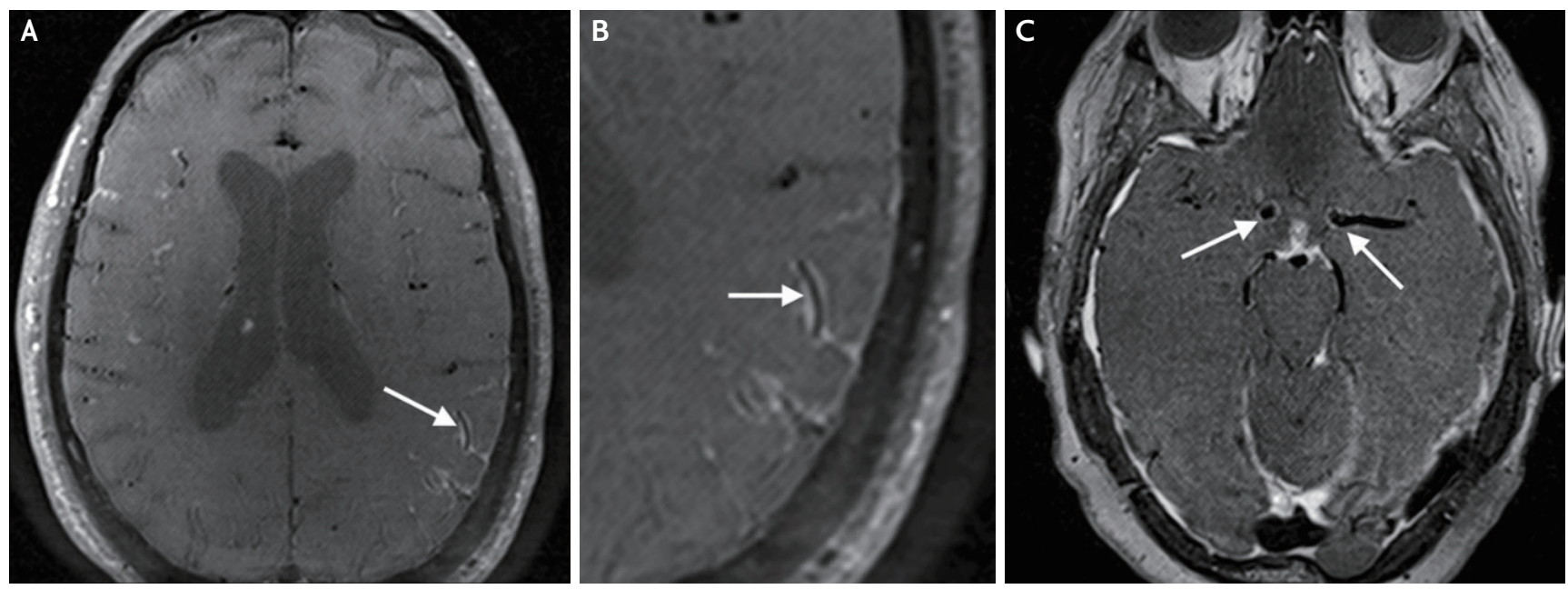

FIG. 4. Vasculitis. (A) 3D axial T1-weighted post-contrast image showing the "tram track" appearance (white arrow) with concentric enhancement from presumed cerebral vasculitis due to inflammatory amyloid angiopathy with adjacent leptomeningeal enhancement from recent superficial siderosis. (B) Higher magnification of the same patient showing the tram track appearance of concentric enhancement (white arrow). (C) $3 \mathrm{D}$ axial T1-weighted post-contrast image in a second patient with bacterial meningitis and associated vasculitis showing the concentric vessel wall enhancement in both terminal internal carotid artery segments (white arrows), which extends along the left middle cerebral artery. 
with distinct vascular territories, a practitioner should suspect vasculitis. The use of vwMRI contributes to the ability to differentiate between ICAD and vasculitis by exposing the "periadventitial" area, which is specific to vasculitis and is defined as enhancement surrounding the vessel wall. ${ }^{42}$

RCVS is characterized by reversible smooth muscle contraction within a vessel wall leading to transient vasoconstriction. It is sometimes referred to as benign cerebral vasculitis because of its similar clinical and radiological findings. ${ }^{24}$ Young and middle-aged women are the population with the highest prevalence. Eclampsia and the postpartum period are associated with a slightly higher incidence. Smoking, alcohol, and certain prescription medication and stimulant drug use have been identified as risk factors. ${ }^{43}$ RCVS may be diagnosed in the presence of sudden-onset headache with and without evidence of concomitant hemorrhage. RCVS may present with alternating constriction and relaxation of the vessel walls throughout the intracranial vasculature, leading to transitory symptoms. Reversibility within three months is considered the most specific feature of RCVS. In contrast to vasculitis, symptoms are highly likely to resolve. The diagnosis is confirmed only after resolution of symptoms. ${ }^{24,44}$

Radiographically, RCVS presents with vessel wall thickening and is usually characterized by the lack of enhancement (Fig. 5). ${ }^{2,24}$ It is presumed that smooth muscle shortening and overlap are the primary causes of observable wall thickening and therefore the vessel wall generally appears isointense. ${ }^{44}$ Occasional atypical radiographic presentations of RCVS demonstrating mildly enhanced vessel walls make its differentiation from CNS vasculitis difficult. The treatment course for RCVS includes elimination of the inciting agent and systemic and/or intra-arterial calcium channel blockers to decrease vascular smooth muscle contraction. It is critical to differentiate RCVS from vasculitis before treatment is carried out, because steroid treatment for a presumed vasculitis will often aggravate the condition if the patient actually has RCVS. ${ }^{45}$

\section{MOYAMOYA}

Moyamoya often appears in the terminal ICA, proximal MCA, or proximal anterior cerebral artery. ${ }^{24} \mathrm{Nar}-$ rowing of the ICA leads to compensatory maturation of the collateral vessels. ${ }^{46}$ The collateral vessels typically display thinner walls, disjointed elastic laminae, and microaneurysms. ${ }^{47}$ Over time, the collateral vessels are known to disappear, shifting some of the burden of blood supply to anastomoses arising from the external carotid artery. ${ }^{4}$ Pathological characteristics of the vessels involved with moyamoya include tunica intima thickening coupled with a diminishing tunica media. The internal elastic lamina is often duplicated and convoluted. In contrast to some other vascular pathologies, wall thickening with moyamoya is not marked by inflammatory or atherosclerotic involvement. ${ }^{47}$ Occlusion within these vessels is tied to the abnormal proliferation of smooth muscle cells and propensity for throm-
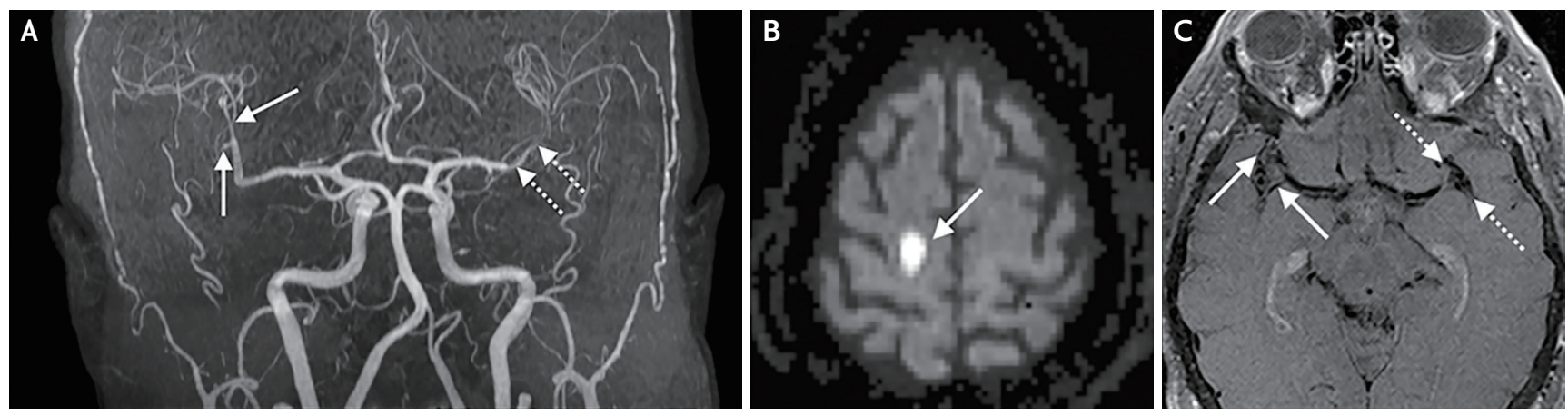

FIG. 5. Reversible cerebral vasoconstriction syndrome. (A) Anterior-posterior digital subtraction angiogram in a patient who presented with post-coital headache and was found to have multifocal luminal narrowing in the bilateral $M_{2}$ branches (dotted and solid white arrows). (B) Axial diffusion-weighted image showing a right hemisphere ischemic stroke (white arrow) in the same patient. (C) $3 \mathrm{D}$ axial T1-weighted post-contrast image showing the absence of vessel wall enhancement at the M2 segments (dotted and solid white arrows), consistent with reversible cerebral vasoconstriction syndrome, which should result in very subtle or no enhancement of the vessel wall at sites of vasospastic narrowing. 
bosis.

Moyamoya progresses through a series of stages that are distinguished by their appearance on imaging, ${ }^{49}$ with DSA representing the primary method of imaging and diagnosis. In cases where differentiating between moyamoya and other diseases such as ICAD is difficult, vwMRI can provide the necessary resolution to aid in diagnosis. ${ }^{24}$ The degree of enhancement seen is notably less than in ICAD, and concentric enhancement patterns are often detected..$^{5}$ The outer diameter of the vessels involved is frequently reduced. Moyamoya is also known to occur bilaterally more often than ICAD.$^{51}$

\section{CONCLUSIONS}

The use of intracranial vwMRI alongside other standard luminal imaging modalities greatly improves the accurate diagnosis, assessment, management, and treatment of patients with cerebrovascular diseases. More research is needed to prospectively understand the pathophysiology of vessel wall inflammation and elucidate its role in the growth and/or rupture of intracranial aneurysms, in intracranial plaque behavior, and in vessel wall hemorrhage in arterial dissection. At present, vwMRI can be used to improve diagnosis and to provide additional information regarding prognosis that can be interpreted in the context of routine demographic, scoring system, and serologic data. Future trials involving this novel imaging modality will likely require the performance of vwMRI at baseline and after 1-2 years to reinforce the findings seen in retrospective studies.

\section{Acknowledgments}

Several of the images used in this article's figures were previously published in an article authored by Dr. de Havenon (Neurovascular Imaging. 2016;2) but have been changed. The creative commons link is: https:// rdcu.be/bMBd4.

\section{Conflicts of Interest}

Dr. Grandhi is a consultant for Medtronic Neurovascular, Cerenovus, and BALT Neurovascular. Dr. Taussky is a consultant for Medtronic Neurovascular, Cerenovus, and Stryker Neurovascular. The other authors report no conflicts of interest.

\section{REFERENCES}

1. Brinjikji W, Mossa-Basha M, Huston J, Rabinstein AA, Lanzino G, Lehman VT. Intracranial vessel wall imaging for evaluation of steno-occlusive diseases and intracranial aneurysms. J Neuroradiol. 2017;44:123-134.

2. Mandell DM, Mossa-Basha M, Qiao Y, Hess CP, Hui F, Matouk C, et al. Intracranial vessel wall MRI: principles and expert consensus recommendations of the American Society of Neuroradiology. AJNR Am J Neuroradiol. 2017;38:218229.

3. Rinkel GJ, Djibuti M, Algra A, van Gijn J. Prevalence and risk of rupture of intracranial aneurysms: a systematic review. Stroke. 1998;29:251-256.

4. Greving JP, Wermer MJ, Brown RD Jr, Morita A, Juvela S, Yonekura M, et al. Development of the PHASES score for prediction of risk of rupture of intracranial aneurysms: a pooled analysis of six prospective cohort studies. Lancet Neurol. 2014;13:59-66.

5. Shiue I, Arima H, Hankey GJ, Anderson CS; ACROSS Group. Modifiable lifestyle behaviours account for most cases of subarachnoid haemorrhage: a population-based case-control study in Australasia. J Neurol Sci. 2012;313:92-94.

6. Qiao Y, Suri FK, Zhang Y, Liu L, Gottesman R, Alonso A, et al. Racial differences in prevalence and risk for intracranial atherosclerosis in a US community-based population. JAMA Cardiol. 2017;2:1341-1348.

7. Backes D, Rinkel GJE, Greving JP, Velthuis BK, Murayama $\mathrm{Y}$, Takao H, et al. ELAPSS score for prediction of risk of growth of unruptured intracranial aneurysms. Neurology. 2017;88:1600-1606.

8. Edjlali M, Gentric JC, Régent-Rodriguez C, Trystram D, Hassen WB, Lion S, et al. Does aneurysmal wall enhancement on vessel wall MRI help to distinguish stable from unstable intracranial aneurysms? Stroke. 2014;45:3704-3706.

9. Austin G, Fisher S, Dickson D, Anderson D, Richardson S. The significance of the extracellular matrix in intracranial aneurysms. Ann Clin Lab Sci. 1993;23:97-105.

10. Chalouhi N, Hoh BL, Hasan D. Review of cerebral aneurysm formation, growth, and rupture. Stroke. 2013;44:36133622 .

11. Texakalidis P, Hilditch CA, Lehman V, Lanzino G, Pereira VM, Brinjikji W. Vessel wall imaging of intracranial aneu- 
rysms: systematic review and meta-analysis. World Neurosurg. 2018;117:453-458.e1.

12. Zhang X, Ares WJ, Taussky P, Ducruet AF, Grandhi R. Role of matrix metalloproteinases in the pathogenesis of intracranial aneurysms. Neurosurg Focus. 2019;47:E4.

13. Portanova A, Hakakian N, Mikulis DJ, Virmani R, Abdalla WM, Wasserman BA. Intracranial vasa vasorum: insights and implications for imaging. Radiology. 2013;267:667-679.

14. Vakil P, Ansari SA, Cantrell CG, Eddleman CS, Dehkordi $\mathrm{FH}$, Vranic J, et al. Quantifying intracranial aneurysm wall permeability for risk assessment using dynamic contrast-enhanced MRI: a pilot study. AJNR Am J Neuroradiol. 2015;36:953-959.

15. Alexander MD, de Havenon A, Kim SE, Parker DL, McNally JS. Assessment of quantitative methods for enhancement measurement on vessel wall magnetic resonance imaging evaluation of intracranial atherosclerosis. Neuroradiology. 2019;61:643-650.

16. Kim JS, Kang DW, Kwon SU. Intracranial atherosclerosis: incidence, diagnosis and treatment. J Clin Neurol. 2005;1:1-7.

17. Khan M, Naqvi I, Bansari A, Kamal AK. Intracranial atherosclerotic disease. Stroke Res Treat. 2011;2011:282845.

18. Inzitari D, Hachinski VC, Taylor DW, Barnett HJ. Racial differences in the anterior circulation in cerebrovascular disease. How much can be explained by risk factors? Arch Neurol. 1990;47:1080-1084.

19. Kawahara I, Morikawa M, Honda M, Kitagawa N, Tsutsumi $\mathrm{K}$, Nagata I, et al. High-resolution magnetic resonance imaging using gadolinium-based contrast agent for atherosclerotic carotid plaque. Surg Neurol. 2007;68:60-65; discussion 65-66.

20. Suemoto CK, Grinberg LT, Leite REP, Ferretti-Rebustini REL, Jacob-Filho W, Yaffe K, et al. Morphometric measurements of extracranial and intracranial atherosclerotic disease: a population-based autopsy study. Atherosclerosis. 2018;270:218-223.

21. Jeng JS, Tang SC, Liu HM. Epidemiology, diagnosis and management of intracranial atherosclerotic disease. Expert Rev Cardiovasc Ther. 2010;8:1423-1432.

22. Nguyen-Huynh MN, Wintermark M, English J, Lam J, Vittinghoff E, Smith WS, et al. How accurate is CT angiography in evaluating intracranial atherosclerotic disease? Stroke. 2008;39:1184-1188.

23. Chen X, Liu Y, Tong H, Dong Y, Ma D, Xu L, et al. Meta-analysis of computed tomography angiography versus magnetic resonance angiography for intracranial aneu- rysm. Medicine (Baltimore). 2018;97:e10771.

24. Choi YJ, Jung SC, Lee DH. Vessel wall imaging of the intracranial and cervical carotid arteries. J Stroke. 2015;17:238-255.

25. Nörenberg D, Ebersberger HU, Diederichs G, Hamm B, Botnar RM, Makowski MR. Molecular magnetic resonance imaging of atherosclerotic vessel wall disease. Eur Radiol. 2016;26:910-920.

26. Chalouhi N, Ali MS, Jabbour PM, Tjoumakaris SI, Gonzalez LF, Rosenwasser RH, et al. Biology of intracranial aneurysms: role of inflammation. J Cereb Blood Flow Metab. 2012;32:1659-1676.

27. Turan TN, Bonilha L, Morgan PS, Adams RJ, Chimowitz MI. Intraplaque hemorrhage in symptomatic intracranial atherosclerotic disease. J Neuroimaging. 2011;21:e159-e161.

28. Xu WH, Li ML, Gao S, Ni J, Yao M, Zhou LX, et al. Middle cerebral artery intraplaque hemorrhage: prevalence and clinical relevance. Ann Neurol. 2012;71:195-198.

29. Yamada N, Higashi M, Otsubo R, Sakuma T, Oyama N, Tanaka R, et al. Association between signal hyperintensity on T1-weighted MR imaging of carotid plaques and ipsilateral ischemic events. AJNR Am J Neuroradiol. 2007;28:287292.

30. Wang GX, Li W, Lei S, Ge XD, Yin JB, Zhang D. Relationships between aneurysmal wall enhancement and conventional risk factors in patients with intracranial aneurysm: a high-resolution MRI study. J Neuroradiol. 2019;46:25-28.

31. McNally JS, McLaughlin MS, Hinckley PJ, Treiman SM, Stoddard GJ, Parker DL, et al. Intraluminal thrombus, intraplaque hemorrhage, plaque thickness, and current smoking optimally predict carotid stroke. Stroke. 2015;46:8490.

32. Hosseini AA, Kandiyil N, Macsweeney ST, Altaf N, Auer DP. Carotid plaque hemorrhage on magnetic resonance imaging strongly predicts recurrent ischemia and stroke. Ann Neurol. 2013;73:774-784.

33. Gupta A, Baradaran H, Schweitzer AD, Kamel H, Pandya A, Delgado D, et al. Carotid plaque MRI and stroke risk: a systematic review and meta-analysis. Stroke. 2013;44:3071-3077.

34. Saam T, Hetterich H, Hoffmann V, Yuan C, Dichgans M, Poppert $\mathrm{H}$, et al. Meta-analysis and systematic review of the predictive value of carotid plaque hemorrhage on cerebrovascular events by magnetic resonance imaging. J Am Coll Cardiol. 2013;62:1081-1091.

35. Zhu C, Tian X, Degnan AJ, Shi Z, Zhang X, Chen L, et al. Clinical significance of intraplaque hemorrhage in lowand high-grade basilar artery stenosis on high-resolution 
MRI. AJNR Am J Neuroradiol. 2018;39:1286-1292.

36. Tan HW, Chen X, Maingard J, Barras CD, Logan C, Thijs V, et al. Intracranial vessel wall imaging with magnetic resonance imaging: current techniques and applications. World Neurosurg. 2018;112:186-198.

37. Li S, Yan B, Kaye A, Mitchell P, Dowling R, Collins M, et al. Prognosis of intracranial dissection relates to site and presenting features. J Clin Neurosci. 2011;18:789-793.

38. Mossa-Basha M, Alexander M, Gaddikeri S, Yuan C, Gandhi D. Vessel wall imaging for intracranial vascular disease evaluation. J Neurointerv Surg. 2016;8:1154-1159.

39. Bonkowsky V, Steinbach S, Arnold W. Vertigo and cranial nerve palsy caused by different forms of spontaneous dissections of internal and vertebral arteries. Eur Arch Otorhinolaryngol. 2002;259:365-368.

40. Ahn SS, Kim BM, Suh SH, Kim DJ, Kim DI, Shin YS, et al. Spontaneous symptomatic intracranial vertebrobasilar dissection: initial and follow-up imaging findings. Radiology. 2012;264:196-202.

41. Sakurai K, Miura T, Sagisaka T, Hattori M, Matsukawa N, Mase M, et al. Evaluation of luminal and vessel wall abnormalities in subacute and other stages of intracranial vertebrobasilar artery dissections using the volume isotropic turbo-spin-echo acquisition (VISTA) sequence: a preliminary study. J Neuroradiol. 2013;40:19-28.

42. Kontzialis M, Wasserman BA. Intracranial vessel wall imaging: current applications and clinical implications. Neurovascular Imaging. 2016;2:4.

43. Ducros A, Boukobza M, Porcher R, Sarov M, Valade D, Bousser MG. The clinical and radiological spectrum of re- versible cerebral vasoconstriction syndrome. a prospective series of 67 patients. Brain. 2007;130:3091-3101.

44. Mandell DM, Matouk CC, Farb RI, Krings T, Agid R, TerBrugge $\mathrm{K}$, et al. Vessel wall MRI to differentiate between reversible cerebral vasoconstriction syndrome and central nervous system vasculitis: preliminary results. Stroke. 2012;43:860-862.

45. French KF, Hoesch RE, Allred J, Wilder M, Smith AG, Digre $\mathrm{KB}$, et al. Repetitive use of intra-arterial verapamil in the treatment of reversible cerebral vasoconstriction syndrome. J Clin Neurosci. 2012;19:174-176.

46. Suzuki J, Takaku A. Cerebrovascular "moyamoya" disease. Disease showing abnormal net-like vessels in base of brain. Arch Neurol. 1969;20:288-299.

47. Achrol AS, Guzman R, Lee M, Steinberg GK. Pathophysiology and genetic factors in moyamoya disease. Neurosurg Focus. 2009;26:E4.

48. Fujimura M, Sonobe S, Nishijima Y, Niizuma K, Sakata $\mathrm{H}$, Kure S, et al. Genetics and biomarkers of moyamoya disease: significance of RNF213 as a susceptibility gene. J Stroke. 2014;16:65-72.

49. Scott RM, Smith ER. Moyamoya disease and moyamoya syndrome. N Engl J Med. 2009;360:1226-1237.

50. Kim YJ, Lee DH, Kwon JY, Kang DW, Suh DC, Kim JS, et al. High resolution MRI difference between moyamoya disease and intracranial atherosclerosis. Eur J Neurol. 2013;20:1311-1318.

51. Ryoo S, Cha J, Kim SJ, Choi JW, Ki CS, Kim KH, et al. High-resolution magnetic resonance wall imaging findings of moyamoya disease. Stroke. 2014;45:2457-2460. 